PROCEEDINGS OF THE AMERICAN MATHEMATICAL SOCIETY

Volume 124, Number 4, April 1996

\title{
FIXED POINTS IN BOUNDARIES OF NEGATIVELY CURVED GROUPS
}

\author{
PHILIP L. BOWERS AND KIM RUANE
}

(Communicated by James E. West)

An important feature of the natural action of a negatively curved (= word hyperbolic) group on its boundary is that the fixed points of hyperbolic (= infinite order) elements form a dense subset of the boundary. Gromov states this in his influential treatise Hyperbolic Groups [4, 8.2 D] and his explanation involves Stalling's theorem on groups with infinitely many ends. This short note provides an elementary proof of this fact. The proof relies on a simple enhancement of the Pumping Lemma from the theory of finite state automata and on the fact that the geodesic words with respect to a fixed generating set for a negatively curved group form a regular language. For background material on negatively curved groups see [1] and on automata and regular languages on groups see [2]. For a different proof see [5].

Definition. Let $L$ be a language over the finite alphabet $A$. An infinite sequence $\left\{a_{i}\right\}$ of letters from $A$ is an $L$-ray provided, for every integer $n>0$, there exists an integer $N \geq n$ such that $a_{1} \cdots a_{N}$ is a word in the language $L$.

The following lemma, which we term an Ultra-Pumping Lemma (UPL), is our aforementioned simple enhancement of the Pumping Lemma.

Ultra-Pumping Lemma. Let $\left\{a_{i}\right\}$ be an $L$-ray where $L$ is a regular language over the finite alphabet $A$. There exists an integer $M \geq 0$ such that, for each integer $n>0$, there exists an integer $N \geq n$ such that $\left(a_{1} \cdots a_{M}\right)\left(a_{M+1} \cdots a_{M+N}\right)^{r} \in L$ for every integer $r \geq 0$.

Proof. Let $W$ be a finite state automaton accepting $L$. The $L$-ray $\left\{a_{i}\right\}$ describes an infinite path in $W$ and, as there are only finitely many (accept) states in $W$ and infinitely many initial segments of $\left\{a_{i}\right\}$ in $L$, this path visits some accept state $s_{\infty}$ infinitely often. Let $M$ be the step at which this path first visits $s_{\infty}$ and choose $N \geq n$ such that $a_{M+1} \cdots a_{M+N}$ describes a loop in $W$ based at $s_{\infty}$.

We use the term $\delta$-negatively curved in the sense of Gromov's $\delta$-hyperbolic condition $[4$, p. 89]. A group $G$ is $\delta$-negatively curved with respect to a finite generating set $A=A^{-1}$ if the corresponding Cayley graph $\Gamma_{A}(G)$ with the usual word metric has a $\delta$-hyperbolic inner product based at the identity; see $[1$, Definition 1.1, p. 8]. A group $G$ is negatively curved (or word hyperbolic) if it is $\delta$-negatively curved with

Received by the editors May 31, 1994.

1991 Mathematics Subject Classification. Primary 20F32.

Key words and phrases. Negatively curved group, Pumping Lemma, boundary of negatively curved group. 
respect to some finite generating set for some $\delta \geq 0$. For an infinite order element $g$ of the negatively curved group $G$, let $g^{ \pm \infty}$ denote the two fixed points of the action of $g$ on $G \cup \partial G$, where $\partial G$ denotes the boundary of $G$. Recall that $g^{ \pm \infty}$ are elements of $\partial G$ and $\lim g^{i}=g^{+\infty}$ and $\lim g^{-i}=g^{-\infty}$.

Theorem $[4,8.2 \mathrm{D}]$. Let $G$ be a negatively curved group with boundary $\partial G$. The set

$$
\left\{g^{ \pm \infty}: g \in G, \operatorname{order}(g)=\infty\right\}
$$

is a dense subset of $\partial G$.

Proof. Recall that $\partial G$ is metrizable [1] and as such sequences suffice to verify denseness. Let $G$ be $\delta$-negatively curved with respect to the finite generating set $A=A^{-1}$ and let $x \in \partial G$. Choose an infinite geodesic path $a_{1} a_{2} \cdots$ based at 1 in the Cayley graph $\Gamma_{A}(G)$ such that $\lim \overline{a_{1} \cdots a_{i}}=x$. Here, $a_{1} \cdots a_{i}$ denotes a word over $A$ and $\overline{a_{1} \cdots a_{i}}$ denotes the element of $G$ obtained by multiplying. The sequence $\left\{a_{i}\right\}$ is an $L$-ray, where $L$ is the language of geodesic words of $G$ with respect to $A$. Recall that $L$ is a regular language [2] and apply the UPL to obtain an integer $M$ and a strictly increasing sequence $0<N(1)<N(2)<\ldots$ of integers such that for all integers $i>0$ and $r \geq 0$,

$$
\left(a_{1} \cdots a_{M}\right)\left(a_{M+1} \cdots a_{M+N(i)}\right)^{r} \in L .
$$

Let $a=a_{1} \cdots a_{M}, b_{i}=a_{M+1} \cdots a_{M+N(i)}$, and $g_{i}=\bar{a} \bar{b}_{i} \bar{a}^{-1}$. Our claim is that the sequence $\left\{g_{i}^{+\infty}\right\}$ converges to $x$. The point is that since $a b_{i}^{r} \in L$ for all integers $r>0$, the path $a b_{i} b_{i} \cdots$, where $b_{i}$ is repeated infinitely, is a geodesic path from 1 to $g_{i}^{+\infty}$ that shares the initial segment $a b_{i}$ of length $M+N(i)$ with the geodesic path $a_{1} a_{2} \cdots$ from 1 to $x$. Since $N(i) \rightarrow \infty$ as $i \rightarrow \infty$, this forces the convergence of $\left\{g_{i}^{+\infty}\right\}$ to $x$. To be precise, the Gromov inner product (extended to the boundary [1]) applied to $x$ and $g_{i}^{+\infty}$ yields

$$
\left(x \cdot g_{i}^{+\infty}\right) \geq M+N(i)-2 \delta
$$

see $\left[1\right.$, Lemma 4.6(4), p. 51]. Hence $\left(x \cdot g_{i}^{+\infty}\right) \rightarrow \infty$ as $i \rightarrow \infty$ and therefore $\left\{g_{i}^{+\infty}\right\}$ converges to $x$ as desired.

Observe that the proof of the theorem yields the following interesting fact: an infinite geodesic path $a_{1} a_{2} \ldots$ based at 1 may be approximated in $\Gamma_{A}(G) \cup \partial G$ by traveling along an initial segment $\left(a_{1} \cdots a_{M}\right)$ and then infinitely often repeating large segments of the path. The UPL and the proof of the theorem may be applied to verify that an infinite quasi-geodesic path may be approximated by traveling on an initial segment of the path and then repeating a subpath, chosen arbitrarily large, infinitely often.

As an application of the theorem, we give a quick proof of a known result.

Corollary. If $H$ is an infinite normal subgroup of the negatively curved group $G$, then the limit set $\Lambda(H)=\partial G \cap \mathrm{Cl}_{G \cup \partial G}(H)$ is equal to $\partial G$.

Proof. Let $x \in \partial G$ and let $U$ be an open neighborhood of $x$ in $\partial G$. Let $h$ be an infinite order element of $H$ [3] and choose an infinite order element $g$ of $G$ such that $g^{+\infty} \in U$. Since $g^{+\infty}$ is an attractive fixed point of the action of $g$ on $G \cup \partial G$ (induced by left multiplication), there is an integer $N>0$ such that $g^{N} \cdot h^{+\infty} \in U$. Since $\lim \left(g^{N} h^{i} g^{-N}\right)=g^{N} \cdot h^{+\infty}$ and $H$ is normal, $g^{N} \cdot h^{+\infty} \in \Lambda(H)$. Since $U$ is an arbitrary neighborhood of $x$, we conclude that $x \in \Lambda(H)$. 


\section{REFERENCES}

1. J.M. Alonso, T. Brady, D. Cooper, V. Ferlini, M. Lustig, M. Mihalik, M. Shapiro, and H. Short (Editor), Notes on word hyperbolic groups, Group Theory from a Geometrical Viewpoint (E. Ghys, A. Haefliger, and A. Verjovsky, eds.), World Scientific, Singapore, 1991, pp. 3-63. MR 93g:57001

2. D.B.A. Epstein, J.W. Cannon, D.F. Holt, S.V.F. Levy, M.S. Paterson, and W.P. Thurston, Word Processing in Groups, Jones and Bartlett, Boston, 1992. MR 93i:20036

3. E. Ghys and P. de la Harpe (eds.), Sur les Groupes Hyperboliques d'après Mikhael Gromov, Progress in Math., vol. 83, Birkhäuser, 1990. MR 92f:53050

4. M. Gromov, Hyperbolic groups, Essays in Group Theory (S.M. Gersten, Editor, ed.), vol. 8, Springer-Verlag, MSRI Publ, 1987, pp. 75-263. MR 89e:20070

5. E. Swenson, Negatively curved groups and related topics, PhD Thesis, Brigham Young University, Provo, Utah (1993).

Department of Mathematics, Florida State University, Tallahassee, Florida 323063027

E-mail address: bowers@math.fsu.edu

E-mail address: ruane@math.fsu.edu 